TRANSACTIONS OF THE

AMERICAN MATHEMATICAL SOCIETY

Volume 360, Number 11, November 2008, Pages 6017-6030

S 0002-9947(08)04439-5

Article electronically published on June 13, 2008

\title{
ON EMBEDDING ALL $n$-MANIFOLDS INTO A SINGLE $(n+1)$-MANIFOLD
}

\author{
FAN DING, SHICHENG WANG, AND JIANGANG YAO
}

\begin{abstract}
For each composite number $n \neq 2^{k}$, there does not exist a single connected closed $(n+1)$-manifold such that any smooth, simply-connected, closed $n$-manifold can be topologically flatly embedded into it. There is a single connected closed 5-manifold $W$ such that any simply-connected, 4-manifold $M$ can be topologically flatly embedded into $W$ if $M$ is either closed and indefinite, or compact and with non-empty boundary.
\end{abstract}

\section{INTRODUCTION AND SOME PREREQUISITES}

The celebrated Whitney embedding theorem states that any smooth $n$-dimensional manifold can be embedded smoothly into the Euclidean space $\mathbb{R}^{2 n}$, or equivalently, any smooth $n$-dimensional manifold can be embedded into any $2 n$-dimensional manifold. It is natural to consider the following general problem:

Find the smallest non-negative integer $e_{n}$, such that any $n$-dimensional connected, closed manifold can be (topologically flatly or smoothly) embedded into a single connected, closed manifold of dimension $n+e_{n}$.

We say that $M$ can be topologically flatly embedded into $W$ if $M \times[-1,1]$ can be topologically embedded into $W$.

Clearly $0 \leq e_{n} \leq n$.

Examples. The only known $e_{i}$ 's are for $i=0,1,2,3$ :

(i) $e_{0}=0$ since a connected 0 -manifold is a point;

(ii) $e_{1}=0$ since a connected, closed 1-manifold is homeomorphic to $S^{1}$;

(iii) $e_{2}=1$ since any orientable, connected, closed surface embeds into $S^{3}$, and any non-orientable, connected, closed surface can be presented as a connected sum of an orientable, connected, closed surface and $\mathbb{R P}^{2}$ or $\mathbb{R P}^{2} \# \mathbb{R} \mathbb{P}^{2}$; hence each connected closed surface can be embedded into $S^{3} \# \mathbb{R} \mathbb{P}^{3} \# \mathbb{R} \mathbb{P}^{3} \cong \mathbb{R} \mathbb{P}^{3} \# \mathbb{R P}^{3}$.

(iv) $e_{3}=2$ since each closed 3-manifold embeds into $S^{5}$, due to Hirsch in the orientable case ([Hi], also [Ki]), and due to Rohlin and Wall independently in the non-orientable case ([$[\mathrm{Ro}$, Wa]; see $[\mathrm{WZ}]$ for a proof using only Dehn surgery). Kawauchi [Ka] showed that there does not exist a single oriented, connected, closed

Received by the editors September 1, 2005 and, in revised form, May 12, 2006 and October 31, 2006.

2000 Mathematics Subject Classification. Primary 57N35.

The authors would like to thank Jianzhong Pan for informing them of Sullivan's work [Su].

The first two authors were partially supported by grant No. 10201003 of NSFC and a grant of MSTC.

(C)2008 American Mathematical Society Reverts to public domain 28 years from publication 
4-manifold such that any connected, closed 3-manifold can be topologically flatly embedded into it by constructing signature invariants from infinite cyclic coverings, and then Shiomi [Shi] showed that Kawauchi's result is still true if the target 4manifold is allowed to be non-orientable.

It is reasonable to guess that, in general, $e_{n}>1$. The main result of the present paper claims that this is the case for "most" $n$, and we prove

Theorem 1.1. If $n$ is a composite number and is not a power of 2 , then there does not exist a connected, closed $(n+1)$-manifold $W$, such that any smooth, simplyconnected, closed n-manifold $M$ can be topologically flatly embedded into $W$.

To prove Theorem 1.1, it is enough to prove there does not exist such an oriented $W$, since if any smooth, simply-connected, closed $n$-manifold $M$ can be topologically flatly embedded into a non-orientable $(n+1)$-manifold $W$, then $M$ can also be topologically flatly embedded into the orientable double cover of $W$ because $M$ is simply-connected.

Besides [Ka] and $\underline{\mathrm{Shi}}$, there are several recent papers ([EL], Fa1], and [Fa2]), which study whether a 3-manifold $M$ embeds into connected sums of $\mathbb{C P}^{2}$. This relates to finding uncountably many smooth structures on the 4-manifold $M \times \mathbb{R}$ (see Di], Fa1 and [Fa2]). Fang's observation (cf. [Fa2]) that there exists an obstruction to the embedding of 3-manifolds into a simply-connected definite 4-manifold was the first inspiration for our proof of Theorem 1.1 .

We do not know if Theorem 1.1 still holds for all remaining positive integers $n \geq 5$. When $n=3$, the only simply-connected, closed 3-manifold is $S^{3}$ if the Poincaré Conjecture is valid. When $n=4$, the situation is still not clear, and we have the following (for terminologies involved, see Section 5).

Theorem 1.2. (a) There exists an oriented, connected, closed 5-manifold $W$, such that for any simply-connected, closed 4-manifold $M$ with indefinite intersection form, $M$ can be topologically flatly embedded into $W$.

(b) Any simply-connected, compact topological 4-manifold $M$ with $\partial M \neq \emptyset$ admits a topologically flat embedding into $S^{2} \tilde{\times} S^{3}$, the non-trivial $S^{3}$-bundle over $S^{2}$.

Remark 1.3. There are indefinite 4-manifolds with arbitrarily large signatures. Theorem 1.2 (a) tells us that the signature of 4-manifolds cannot be an obstruction for our codimension 1 embeddings. Note that any oriented, connected, closed 4manifold with non-zero signature does not bound a compact orientable 5-manifold. It follows that the first Betti number of $W$ in (a) must be positive and that the condition $\partial M \neq \emptyset$ in (b) cannot be removed.

\section{The organization of the paper and an outline of the proofs.}

Roughly speaking, we prove Theorem 1.1 as follows. First assume that there exists such a manifold $W$. In Proposition 2.1, we produce a function $\varphi$ for each embedding $M^{n} \rightarrow W$. Proposition 3.3 shows that cup products admitting such a function $\varphi$ are rare. Then we construct an $M^{n}$ whose cup product doesn't admit such a function $\varphi$, thus get a contradiction.

In Section 2, for any fixed $(n+1)$-manifold $W$, Proposition 2.1 claims that for each topologically flat codimension 1 embedding $M^{n} \rightarrow W$ and each factorization $n=$ $p q$, there is a homological obstruction to the embedding in terms of $H^{p+1}(W ; \mathbb{R})$, $H^{p}(M ; \mathbb{R})$, the $q$-multiple cup product on $H^{p}(M ; \mathbb{R})$, and so on. Since $n$ is not a 
prime number and is not a power of 2 , we can assume that the factorization $n=p q$ has been chosen so that $p \geq 2$ and $q \geq 3$, and $q$ is odd.

Let $\left(\wedge^{q} \mathbb{R}^{m}\right)^{*}\left(\right.$ resp. $\left.\left(\vee^{q} \mathbb{R}^{m}\right)^{*}\right)$ be the vector space of all skew-symmetric (resp. symmetric) $q$-multilinear functions on the $m$-dimensional real vector space $\mathbb{R}^{m}$. In Section 3 we study special (see Definition in Section 3) functions in $\left(\wedge^{q} \mathbb{R}^{m}\right)^{*}$ (resp. $\left.\left(\vee^{q} \mathbb{R}^{m}\right)^{*}\right)$, an example of which is the $q$-multiple cup product on $H^{p}(M ; \mathbb{R})$ appearing in Proposition 2.1. Proposition 3.3 says that, when $q \geq 3$ is odd and $m$ is sufficiently large, such functions are contained in a proper closed subset of $\left(\wedge^{q} \mathbb{R}^{m}\right)^{*}\left(\right.$ resp. $\left.\left(\vee^{q} \mathbb{R}^{m}\right)^{*}\right)$.

In Section 4 we first choose a suitable rational non-special function provided by Proposition 3.3, and then Proposition 4.2 claims that there is a commutative graded algebra $A=\bigoplus_{i=0}^{q} A_{i p}$ over $\mathbb{Q}$ satisfying Poincaré duality so that the chosen non-special function is the $q$-multiple cup product on $A_{p}$. Next we verify that $A$ can be realized as the rational cohomology ring of a simply-connected closed $n$-manifold $M$ by invoking a celebrated result of Sullivan. Hence the $q$-multiple cup product on $H^{p}(M ; \mathbb{R})$ is a non-special function, and therefore $M$ cannot be topologically flatly embedded into $W$ by Proposition 2.1, which finishes the proof of Theorem 1.1.

Section 5 is devoted to the case $n=4$, and we get Theorem 1.2 by explicit constructions and the classification theorem for simply-connected, closed 4-manifolds.

In the remainder of this section, we recall and fix needed notation and conventions in tensor algebra based on $\underline{\mathrm{Sh}}$.

Prerequisites on tensor algebra. Let $V$ be an $m$-dimensional vector space over a field $\mathbb{F}$ of characteristic zero. Denote by $\otimes^{p} V$ the $p$-fold tensor product of $V$, and by $\otimes V=\bigoplus_{p}\left(\otimes^{p} V\right)$ the tensor algebra.

The $p$ th exterior power of $V$ is denoted by $\wedge^{p} V$. The product operation $\wedge$ : $\left(\wedge^{p} V\right) \times\left(\wedge^{q} V\right) \rightarrow \wedge^{p+q} V$ can be extended bilinearly to general elements of the vector space $\wedge V=\bigoplus_{p}\left(\wedge^{p} V\right)=\mathbb{F} \oplus V \oplus \wedge^{2} V \oplus \ldots \oplus \wedge^{m} V$ to make $\wedge V$ into an algebra over $\mathbb{F}$, called the exterior algebra on $V$.

Similarly the $p$ th symmetric power of $V$ is denoted by $\vee^{p} V$. The product operation $\vee:\left(\vee^{p} V\right) \times\left(\vee^{q} V\right) \rightarrow \vee^{p+q} V$ can be extended bilinearly to general elements of the vector space $\vee V=\bigoplus_{p}\left(\vee^{p} V\right)=\mathbb{F} \oplus V \oplus \vee^{2} V \oplus \ldots$ to make $\vee V$ into an infinite-dimensional algebra over $\mathbb{F}$, called the symmetric algebra on $V$.

Let $\times^{p} V$ denote the Cartesian product $V \times V \times \ldots \times V$ of $p$ copies of $V$. Each multilinear function $F: \times^{p} V \rightarrow \mathbb{F}$ determines a unique linear function $L: \otimes^{p} V \rightarrow \mathbb{F}$ such that for all $v_{1}, \ldots, v_{p} \in V, L\left(v_{1} \otimes \ldots \otimes v_{p}\right)=F\left(v_{1}, \ldots, v_{p}\right)$. The correspondence $F \leftrightarrow L$ establishes a natural isomorphism between the vector space $L\left(\times{ }^{p} V ; \mathbb{F}\right)$ of all multilinear functions and the dual space $\left(\otimes^{p} V\right)^{*}$ of $\otimes^{p} V$. For any skewsymmetric (resp. symmetric) multilinear function $F: \times^{p} V \rightarrow \mathbb{F}$, there exists a unique linear function $L_{s}: \wedge^{p} V\left(\right.$ resp. $\left.\vee^{p} V\right) \rightarrow \mathbb{F}$ such that for all $v_{1}, \ldots, v_{p} \in V$, $L_{s}\left(v_{1} \wedge \ldots \wedge v_{p}\right)=F\left(v_{1}, \ldots, v_{p}\right)\left(\right.$ resp. $\left.L_{s}\left(v_{1} \vee \ldots \vee v_{p}\right)=F\left(v_{1}, \ldots, v_{p}\right)\right)$. The correspondence $F \leftrightarrow L_{s}$ establishes a natural isomorphism between the vector space $\operatorname{Sk}\left(\times^{p} V ; \mathbb{F}\right)$ (resp. $\left.\operatorname{Sym}\left(\times^{p} V ; \mathbb{F}\right)\right)$ of all skew-symmetric (resp. symmetric) multilinear functions and the dual space $\left(\wedge^{p} V\right)^{*}$ (resp. $\left.\left(\vee^{p} V\right)^{*}\right)$ of $\wedge^{p} V$ (resp. $\vee^{p} V$ ). Thus we may consider a skew-symmetric (resp. symmetric) multilinear function $F: \times^{p} V \rightarrow \mathbb{F}$ as an element of $\left(\wedge^{p} V\right)^{*}$ (resp. $\left.\left(\vee^{p} V\right)^{*}\right)$, and vice versa.

Let $\left\{e_{1}, \ldots, e_{m}\right\}$ be the standard basis for the real vector space $\mathbb{R}^{m}$; i.e. $e_{i}=$ $(0, \ldots, 0,1,0, \ldots, 0)$ has components 0 except for its $i$-th component, which is equal to 1. Each element $F \in\left(\wedge^{q} \mathbb{R}^{m}\right)^{*}\left(\right.$ resp. $\left.\left(\vee^{q} \mathbb{R}^{m}\right)^{*}\right)$ is determined by $\left(\begin{array}{c}m \\ q\end{array}\right)$ (resp. 
$(\underset{q}{m+q-1}))$ real numbers $\left(F_{i_{1} \ldots i_{q}}\right)$, where $F_{i_{1} \ldots i_{q}}=F\left(e_{i_{1}}, \ldots, e_{i_{q}}\right)$ for $1 \leq i_{1}<$ $\ldots<i_{q} \leq m$ (resp. $\left.1 \leq i_{1} \leq \ldots \leq i_{q} \leq m\right)$. Thus $\operatorname{dim}\left(\wedge^{q} \mathbb{R}^{m}\right)^{*}=\left(\begin{array}{c}m \\ q\end{array}\right)$ and $\left(\vee^{q} \mathbb{R}^{m}\right)^{*}=\left(\begin{array}{c}m+q-1 \\ q\end{array}\right)$.

\section{Homological obstruCtions FOR EMBEDdings}

From now on all manifolds appearing in this paper are considered to be oriented and connected. $\beta_{i}(X)$ denotes the $i$-th Betti number of a compact manifold $X$.

The following proposition sets up a property on the cohomology ring of the $n$-manifold $M$ if it can be embedded into the $(n+1)$-manifold $W$.

Proposition 2.1. Let $M^{n}$ and $W^{n+1}$ be closed and connected, oriented manifolds.

If $M^{n}$ topologically flatly embeds in $W^{n+1}$, then for any integer factorization $n=p q$, where $p, q>0$, there exists a subspace $V$ of $H^{p}(M ; \mathbb{R})$ and a linear transformation $\varphi: V \rightarrow H^{p}(M ; \mathbb{R})$ such that

(i) $\varphi$ has no fixed non-zero vectors,

(ii) $\operatorname{dim} V \geq \frac{1}{2}\left(\beta_{p}(M)-\beta_{p+1}(W)\right)$, and

(iii) for any $x_{1}, \ldots, x_{q} \in V, x_{1} \cup \ldots \cup x_{q}=\varphi\left(x_{1}\right) \cup \ldots \cup \varphi\left(x_{q}\right)$.

Proof. All homology and cohomology groups in this proof are with coefficients in $\mathbb{R}$. We divide the proof into two cases.

From the definition of a topologically flat embedding, we can identify a closed tubular neighborhood of $M$ in $W$ with $M \times[-1,1]$.

Case 1. Suppose $W \backslash M$ is not connected. Denote the closures of the two components of $W \backslash M$ by $W_{1}^{\prime}$ and $W_{2}^{\prime}$, and let $W_{i}$ be the union of $W_{i}^{\prime}$ and $M \times(-1,1)$. Then $\left\{W_{1}, W_{2},\right\}$ is an excisive couple and from the Mayer-Vietoris sequence, we obtain $H^{p}\left(W_{1}\right) \oplus H^{p}\left(W_{2}\right) \stackrel{f}{\rightarrow} H^{p}(M) \stackrel{\delta^{*}}{\rightarrow} H^{p+1}(W)$. Here the first map is given by $f\left(w_{1}, w_{2}\right)=i_{1}^{*}\left(w_{1}\right)-i_{2}^{*}\left(w_{2}\right)$ for $w_{1} \in H^{p}\left(W_{1}\right), w_{2} \in H^{p}\left(W_{2}\right)$, where $i_{1}$ and $i_{2}$ are inclusions $M \hookrightarrow W_{1}$ and $M \hookrightarrow W_{2}$ respectively. Clearly $\operatorname{dim} \operatorname{im}(f)=\operatorname{dim} \operatorname{ker}\left(\delta^{*}\right) \geq$ $\operatorname{dim} H^{p}(M)-\operatorname{dim} H^{p+1}(W)=\beta_{p}(M)-\beta_{p+1}(W) . \operatorname{Since} \operatorname{dim} \operatorname{im}\left(i_{1}^{*}\right)+\operatorname{dimim}\left(i_{2}^{*}\right) \geq$ $\operatorname{dimim}(f)$, we may set $V=\operatorname{im}\left(i_{1}^{*}\right)$ and assume that $\operatorname{dim} V \geq \frac{1}{2}\left(\beta_{p}(M)-\beta_{p+1}(W)\right)$.

For any $x_{1}, \ldots, x_{q} \in V$, there exist $y_{1}, \ldots, y_{q} \in H^{p}\left(W_{1}\right)$ such that $x_{i}=i_{1}^{*}\left(y_{i}\right), 1 \leq$ $i \leq q$. Since $M=\partial W_{1}^{\prime}$, we have $i_{1 *}[M]=0 \in H_{n}\left(W_{1}\right)$, where $[M]$ denotes the fundamental class of $M$. So $\left\langle x_{1} \cup \ldots \cup x_{q},[M]\right\rangle=\left\langle i_{1}^{*}\left(y_{1} \cup \ldots \cup y_{q}\right),[M]\right\rangle=$ $\left\langle y_{1} \cup \ldots \cup y_{q}, i_{1 *}[M]\right\rangle=0$. Therefore for any $x_{1}, \ldots, x_{q} \in V, x_{1} \cup \ldots \cup x_{q}=0$.

The result is thus proved by letting $\varphi$ be the zero map.

Case 2. Suppose $W \backslash M$ is connected. Let $W_{1}=M \times(-1,1)$ and $W_{2}=$ $W \backslash\left(M \times\left[-\frac{1}{2}, \frac{1}{2}\right]\right)$. Clearly, $W_{1} \cap W_{2}=M \times\left(-1,-\frac{1}{2}\right) \cup M \times\left(\frac{1}{2}, 1\right)$.

Again by the Mayer-Vietoris sequence, we get the exact sequence $H^{p}\left(W_{1}\right) \oplus H^{p}\left(W_{2}\right) \rightarrow H^{p}\left(W_{1} \cap W_{2}\right) \rightarrow H^{p+1}(W)$, which reduces to $H^{p}(M) \oplus$ $H^{p}\left(W_{2}\right) \stackrel{f}{\rightarrow} H^{p}(M) \oplus H^{p}(M) \stackrel{\delta^{*}}{\rightarrow} H^{p+1}(W)$. Now $f$ can be written as $f(x, w)=$ $\left(x-i_{0}^{*}(w), x-i_{1}^{*}(w)\right)$, where $i_{0}$ and $i_{1}$ are inclusions $M \times\{-1\} \hookrightarrow W_{2}$ and $M \times\{1\} \hookrightarrow W_{2}$, respectively. By the exactness, $\operatorname{dim} \operatorname{im}(f)=\operatorname{dim} \operatorname{ker}\left(\delta^{*}\right) \geq$ $\operatorname{dim}\left(H^{p}(M) \oplus H^{p}(M)\right)-\operatorname{dim} H^{p+1}(W)=2 \beta_{p}(M)-\beta_{p+1}(W)$.

Let $\Delta=\left\{(x, x) \mid x \in H^{p}(M)\right\} \subset H^{p}(M) \oplus H^{p}(M)$ and $U=\left\{\left(i_{0}^{*}(w), i_{1}^{*}(w)\right) \mid w \in\right.$ $\left.H^{p}\left(W_{2}\right)\right\}$. Then $\Delta+U=\operatorname{im}(f)$ and therefore $\operatorname{dim}(\Delta+U)=\operatorname{dim} \operatorname{im}(f) \geq 2 \beta_{p}(M)-$ $\beta_{p+1}(W)$. Since $\operatorname{dim} \Delta=\beta_{p}(M)$, there is a subspace $S$ of $U$ with $\operatorname{dim} S \geq \beta_{p}(M)-$ $\beta_{p+1}(W)$ such that $S \cap \Delta=\{(0,0)\}$. Let $\pi_{1}, \pi_{2}: H^{p}(M) \oplus H^{p}(M) \rightarrow H^{p}(M)$ be the projections of $H^{p}(M) \oplus H^{p}(M)$ onto its first and second factors, respectively. 
Since $\operatorname{dim} \pi_{1}(S)+\operatorname{dim} \pi_{2}(S) \geq \operatorname{dim} S$, without loss of generality, we may assume that $\operatorname{dim} \pi_{1}(S) \geq \frac{1}{2} \operatorname{dim} S \geq \frac{1}{2}\left(\beta_{p}(M)-\beta_{p+1}(W)\right)$.

It follows from $\partial\left(W_{2} \backslash M \times(-1,1)\right)=-(M \times\{-1\}) \cup(M \times\{1\})$ that $i_{0 *}[M]=$ $i_{1 *}[M]$. Thus for any $w_{1}, \ldots, w_{q} \in H^{p}\left(W_{2}\right),\left\langle w_{1} \cup \ldots \cup w_{q}, i_{0 *}[M]-i_{1 *}[M]\right\rangle=0$, and this implies $\left\langle i_{0}^{*}\left(w_{1}\right) \cup \ldots \cup i_{0}^{*}\left(w_{q}\right),[M]\right\rangle=\left\langle i_{1}^{*}\left(w_{1}\right) \cup \ldots \cup i_{1}^{*}\left(w_{q}\right),[M]\right\rangle$. In particular, if $\left(u_{1}, v_{1}\right), \ldots,\left(u_{q}, v_{q}\right) \in S$, then

$$
u_{1} \cup \ldots \cup u_{q}=v_{1} \cup \ldots \cup v_{q} .
$$

Let $\left\{y_{1}, \ldots, y_{r}\right\}$ be a basis for $\pi_{1}(S)$. We can find $z_{1}, \ldots, z_{r} \in H^{p}(M)$ such that $\left(y_{j}, z_{j}\right) \in S, 1 \leq j \leq r$. Then there is a unique linear transformation $\varphi: \pi_{1}(S) \rightarrow$ $H^{p}(M)$ such that $\varphi\left(y_{j}\right)=z_{j}, 1 \leq j \leq r$.

One can write any $y \in \pi_{1}(S)$ as $y=\sum_{j} c_{j} y_{j}$. Then $\varphi(y)=\sum_{j} c_{j} z_{j}$. Thus $(y, \varphi(y)) \in S$. Since $S \cap \Delta=\{(0,0)\}$, if $y \neq 0$, then $\varphi(y) \neq y$. Now setting $V=\pi_{1}(S)$, one gets the desired result from equation (2.1).

\section{Special multilinear functions}

Proposition 2.1 motivates the following definition.

Definition 3.1. Let $F$ be an element of $\left(\wedge^{q} \mathbb{R}^{m}\right)^{*}\left(\operatorname{resp} .\left(\vee^{q} \mathbb{R}^{m}\right)^{*}\right)$. We say that $F$ is special if there exist a subspace $U$ of $\mathbb{R}^{m}$ with $\operatorname{dim} U \geq \frac{m}{3}$ and a linear map $\varphi: U \rightarrow \mathbb{R}^{m}$ with no fixed non-zero vectors such that for all $x_{1}, \ldots, x_{q} \in U$, $F\left(x_{1}, \ldots, x_{q}\right)=F\left(\varphi\left(x_{1}\right), \ldots, \varphi\left(x_{q}\right)\right)$. In the equation, we consider $F$ as a skewsymmetric (resp. symmetric) multilinear function (cf. Section 11).

Example 3.2. Suppose $M^{n}$ topologically flatly embeds in $W^{n+1}, n=p q, p, q>0$, and $\beta_{p}(M) \geq 3 \beta_{p+1}(W)$. Then by Proposition 2.1, the $q$-multiple cup product on $H^{p}(M, \mathbb{R})$ is special.

In this section, we will show

Proposition 3.3. Suppose $q \geq 3$ is an odd integer. If $m$ is sufficiently large, then there exists a proper closed subset $X_{m}$ of $\left(\wedge^{q} \mathbb{R}^{m}\right)^{*}\left(\right.$ resp. $\left.\left(\vee^{q} \mathbb{R}^{m}\right)^{*}\right)$ such that if $F \in\left(\wedge^{q} \mathbb{R}^{m}\right)^{*}$ (resp. $\left.F \in\left(\vee^{q} \mathbb{R}^{m}\right)^{*}\right)$ is special, then $F \in X_{m}$.

Remark 3.4. If $q \geq 2$ is even, then each $F \in\left(\wedge^{q} \mathbb{R}^{m}\right)^{*}\left(\right.$ resp. $\left.F \in\left(\vee^{q} \mathbb{R}^{m}\right)^{*}\right)$ is special. We may simply take $U=\mathbb{R}^{m}$ and $\varphi: \mathbb{R}^{m} \rightarrow \mathbb{R}^{m}$ to be the map sending $x$ to $-x$ for all $x \in \mathbb{R}^{m}$.

Before proving Proposition 3.3. we shall discuss two useful lemmas.

It is a classical result about the Jordan normal form that (cf. Theorem 3.4.5 in [HJ]) any real square matrix is similar to a block diagonal matrix, where each block takes one of the following two forms:

$$
J_{k}(a)=\left[\begin{array}{cccc}
a & 1 & & \\
& \ddots & \ddots & \\
& & \ddots & 1 \\
& & & a
\end{array}\right], C_{l}(a, b)=\left[\begin{array}{ccccccc}
a & b & 1 & 0 & & & \\
-b & a & 0 & 1 & & & \\
& & \ddots & & \ddots & & \\
& & & & & 1 & 0 \\
& & & & \ddots & 0 & 1 \\
& & & & a & b \\
& & & & & -b & a
\end{array}\right] .
$$


The sizes of these two square matrices are $k$ and $2 l$ respectively, where $k$ and $l$ are positive integers, $a$ and $b \neq 0$ are real numbers.

We sort the blocks described above into 6 forms: 1: $J_{1}(1), \mathbf{2 :} J_{1}(-1), \mathbf{3 :} J_{1}(a)$, $|a|>1, \quad 4: J_{1}(a),|a|<1, \mathbf{5 :} J_{k}(a), k>1, \quad \mathbf{6}: C_{l}(a, b)$. By using the Jordan form, we can find a suitable basis for $\mathbb{R}^{m}$ under which the linear map $\varphi$ mentioned in the above definition has a simple matrix representation.

We use $\operatorname{span}\left\{v_{1}, \ldots, v_{k}\right\}$ to denote the subspace of $\mathbb{R}^{m}$ generated by vectors $v_{1}, \ldots, v_{k} \in \mathbb{R}^{m}$, and $\lfloor c\rfloor$ to denote the greatest integer $\leq c$ for $c \in \mathbb{R}$.

Lemma 3.5. Let $U$ be a subspace of $\mathbb{R}^{m}, \varphi: U \rightarrow \mathbb{R}^{m}$ be a linear map with no fixed non-zero vectors, and $l=\left\lfloor\frac{\operatorname{dim} U}{18}\right\rfloor$.

There exists a basis $\left\{v_{1}, \ldots, v_{m}\right\}$ for $\mathbb{R}^{m}$ such that $V=\operatorname{span}\left\{v_{1}, \ldots, v_{l}\right\}$ is a subspace of $U$ and the matrix of $\varphi \mid V$ with respect to this basis is $\left[\begin{array}{l}\Lambda \\ \Gamma\end{array}\right]$, where $\Lambda=$ $\operatorname{diag}\left(\lambda_{1}, \ldots, \lambda_{l}\right)$ is a diagonal matrix with $\lambda_{i_{1}} \ldots \lambda_{i_{q}} \neq 1$ for any odd integer $q$ and $1 \leq i_{1} \leq \ldots \leq i_{q} \leq l$.

Proof. Choose a basis $\left\{u_{1}, \ldots, u_{k}\right\}$ for $U$ and complete it to a basis $\left\{u_{1}, \ldots, u_{m}\right\}$ for $\mathbb{R}^{m}$, where $k=\operatorname{dim} U$. Denote the matrix of $\varphi$ with respect to this basis by $\left[\begin{array}{l}J \\ *\end{array}\right]$, where $J$ is a $k \times k$ square matrix. We may choose $\left\{u_{1}, \ldots, u_{k}\right\}$ so that $J$ is in real Jordan canonical form. The Jordan blocks have 6 forms as mentioned above. For some $i_{0}$, the total size $t$ of blocks of form $i_{0}$ in $J$ should satisfy $t \geq \frac{k}{6}$. We change the order of $u_{1}, \ldots, u_{k}$ so that in the block expression for $J$, blocks of form $i_{0}$ appear first.

Suppose $i_{0}=2,3$ or 4 . Choose $\left\{v_{1}, \ldots, v_{m}\right\}$ to be just $\left\{u_{1}, \ldots, u_{m}\right\}$. For the matrix representation $\left[\begin{array}{l}\Lambda \\ \Gamma\end{array}\right]$ of $\varphi \mid V$ under this basis, as $l \leq t$ and each Jordan block of form $i_{0}$ is a $1 \times 1$ matrix, $\Lambda$ is a diagonal matrix, $\operatorname{diag}\left(\lambda_{1}, \ldots, \lambda_{l}\right)$. For any $1 \leq i_{1} \leq \ldots \leq i_{q} \leq l$, if $i_{0}=2$, then $\lambda_{i_{1}} \ldots \lambda_{i_{q}}=-1$ since $q$ is odd; if $i_{0}=3$, then $\left|\lambda_{i_{1}} \ldots \lambda_{i_{q}}\right|>1$; and if $i_{0}=4$, then $\left|\lambda_{i_{1}} \ldots \lambda_{i_{q}}\right|<1$. In any case, $\lambda_{i_{1}} \ldots \lambda_{i_{q}} \neq 1$.

Suppose $i_{0}=1,5$ or 6 . If there exists a subspace $V$ of $U$ such that

$$
\operatorname{dim} V=l \text { and } \varphi(V) \cap V=\{0\},
$$

then we can choose a basis $\left\{v_{1}, \ldots, v_{l}\right\}$ for $V$, a basis for $\varphi(V)$, and extend the union of these two bases to a basis $\left\{v_{1}, \ldots, v_{m}\right\}$ for $\mathbb{R}^{m}$. As $\varphi(V) \cap V=\{0\}$, the matrix representation for $\varphi \mid V$ must be $\left[\begin{array}{l}0 \\ \Gamma\end{array}\right]$, which satisfies the requirement.

Now we only need to find a subspace $V$ with property (3.1). Set $U^{\prime}=$ $\operatorname{span}\left\{u_{1}, \ldots, u_{t}\right\}$ and denote the matrix of $\varphi \mid U^{\prime}$ with respect to the basis $\left\{u_{1}, \ldots, u_{m}\right\}$ by $\left[\begin{array}{c}J_{0} \\ *\end{array}\right]$.

Suppose first that $J_{0}$ contains only blocks of the same form $J_{1}(1)$. Let $W=$ $\operatorname{span}\left\{u_{t+1}, \ldots, u_{m}\right\}$. Then $\mathbb{R}^{m}=U^{\prime} \oplus W$. Since $J_{0}=I$, for $u \in U^{\prime}, \varphi(u)=u+w$ for some $w \in W$. Suppose that $x \in \varphi\left(U^{\prime}\right) \cap U^{\prime}$. Then $x \in U^{\prime}$ and there exists $u \in U^{\prime}$ such that $\varphi(u)=x$. But $\varphi(u)=u+w=x+0$, so $\varphi(u)=u$, and hence $u=0$, since $\varphi$ only fixes the zero vector. Therefore $\varphi\left(U^{\prime}\right) \cap U^{\prime}=\{0\}$, and we may simply shrink $U^{\prime}$ to $V$ with $\operatorname{dim} V=l$ as $t \geq l$.

Suppose now $J_{0}$ contains only blocks of the same forms $J_{k}(a), k>1$, or $C_{l}(a, b)$. Fix a block $B_{j}$, let $\left\{w_{1}, \ldots, w_{k_{j}}\right\}$ be the subset of the basis $\left\{u_{1}, \ldots, u_{t}\right\}$ that 
corresponds to the block $B_{j}$. Note that $k_{j} \geq 2$. Set $W_{j}=\operatorname{span}\left\{w_{1}, \ldots, w_{k_{j}}\right\}$, $W_{j, 0}=\operatorname{span}\left\{w_{i} \mid i\right.$ even $\}$ and $W_{j, 1}=\operatorname{span}\left\{w_{i} \mid i\right.$ odd $\}$. Clearly, $W_{j}=W_{j, 0} \oplus W_{j, 1}$ and $\operatorname{dim} W_{j, 0} \geq \frac{k_{j}}{3}$.

Denote the projection of $\mathbb{R}^{m}$ onto $W_{j, 1}$ by $p_{1}$. (This projection is well defined as there is a natural decomposition $\mathbb{R}^{m}=W_{j} \oplus W^{\prime}$, where $W^{\prime}=$ $\operatorname{span}\left(\left\{u_{1}, \ldots, u_{m}\right\} \backslash\left\{w_{1}, \ldots, w_{k_{j}}\right\}\right)$.) If $B_{j}=C_{\frac{k_{j}}{2}}(a, b)$, where $k_{j}$ is necessarily even, then $p_{1} \varphi\left(w_{2 i}\right)=b w_{2 i-1}, 1 \leq i \leq \frac{k_{j}}{2}$, and if $B_{j}=J_{k_{j}}(a)$, then $p_{1} \varphi\left(w_{2 i}\right)=$ $w_{2 i-1}, 1 \leq i \leq\left\lfloor\frac{k_{j}}{2}\right\rfloor$. By these two equations, we know $\varphi\left(W_{j, 0}\right) \cap W_{j, 0}=\{0\}$. Similarly, if we take $V^{\prime}$ to be the direct sum $\bigoplus_{j} W_{j, 0}$ for all blocks in $J_{0}$, we will have $\varphi\left(V^{\prime}\right) \cap V^{\prime}=\{0\}$ and $\operatorname{dim} V^{\prime} \geq \frac{t}{3} \geq \frac{\operatorname{dim} U}{18}$. We may simply shrink $V^{\prime}$ to $V$ with $\operatorname{dim} V=l$.

The next lemma uses the transcendence degree of a field extension, which follows from Chap. VIII, Theorem 1.1 of [La].

Lemma 3.6. Let $g_{1}\left(x_{1}, \ldots, x_{r}\right), \ldots, g_{s}\left(x_{1}, \ldots, x_{r}\right)$ be rational functions in $r$ variables $x_{1}, \ldots, x_{r}$ with coefficients in $\mathbb{R}$. If $s>r$, then there exists a non-zero polynomial $P$ in $s$ variables such that $P\left(g_{1}\left(x_{1} \ldots, x_{r}\right), \ldots, g_{s}\left(x_{1}, \ldots, x_{r}\right)\right)=0$.

Proof of Proposition 3.3. Suppose that $F \in\left(\wedge^{q} \mathbb{R}^{m}\right)^{*}$ is special. By definition, there exists a subspace $U$ of $\mathbb{R}^{m}$ with $\operatorname{dim} U \geq \frac{m}{3}$ and a linear map $\varphi: U \rightarrow \mathbb{R}^{m}$ with no fixed non-zero vectors such that for all $x_{1}, \ldots, x_{q} \in U$,

$$
F\left(x_{1}, \ldots, x_{q}\right)=F\left(\varphi\left(x_{1}\right), \ldots, \varphi\left(x_{q}\right)\right) .
$$

The strategy for the proof is roughly as follows. We will use equation (3.2) to express the coordinates of $F$ in the standard basis for $\left(\wedge^{q} \mathbb{R}^{m}\right)^{*}$. These coordinates will be expressed as rational functions of some variables, and the number of variables used will be less than $\operatorname{dim}\left(\wedge^{q} \mathbb{R}^{m}\right)^{*}$ when $m$ is large enough. Thus according to Lemma 3.6. all special $F$ lie in the zero set of a single non-zero polynomial, which is a proper closed subset of $\left(\wedge^{q} \mathbb{R}^{m}\right)^{*}$.

For the subspace $U$ and the linear map $\varphi$, choose a basis $\left\{v_{1}, \ldots, v_{m}\right\}$ for $\mathbb{R}^{m}$ with the properties described in Lemma 3.5. Let $\left\{e_{1}, \ldots, e_{m}\right\}$ be the standard basis for $\mathbb{R}^{m}$. Set $v_{j}=\sum_{i=1}^{m} \tau_{i j} e_{i}$, define $F_{i_{1} \ldots i_{q}}=F\left(e_{i_{1}}, \ldots, e_{i_{q}}\right)$ and $f_{i_{1} \ldots i_{q}}=$ $F\left(v_{i_{1}}, \ldots, v_{i_{q}}\right), 1 \leq i_{1}<\ldots<i_{q} \leq m$. Clearly, each $F_{i_{1} \ldots i_{q}}$ is a rational function of the variables $\tau_{i j}$ and $f_{j_{1} \ldots j_{q}}$.

Obviously, Lemma 3.5 still holds for any integer $l$ less than $\left\lfloor\frac{\operatorname{dim} U}{18}\right\rfloor$. Now $l=$ $\left\lfloor\frac{\operatorname{dim} U}{18}\right\rfloor \geq\left\lfloor\frac{m}{54}\right\rfloor$, and here we always set $l=\left\lfloor\frac{m}{54}\right\rfloor$. Recall that $V=\operatorname{span}\left\{v_{1}, \ldots, v_{l}\right\}$ $\subset U$, and the matrix of $\varphi \mid V$ with respect to the basis $\left\{v_{1}, \ldots, v_{m}\right\}$ is $\left[\begin{array}{l}\Lambda \\ \Gamma\end{array}\right]$, where $\Lambda=\operatorname{diag}\left(\lambda_{1}, \ldots, \lambda_{l}\right)$ and $\Gamma=\left(\gamma_{i j}\right)_{(m-l) \times l}$.

Define $R=\left\{\left(i_{1}, \ldots, i_{q}\right) \mid 1 \leq i_{1}<\ldots<i_{q} \leq m\right\}, R_{1}=\left\{\left(i_{1}, \ldots, i_{q}\right) \mid 1 \leq i_{1}<\right.$ $\left.\ldots<i_{q} \leq l\right\}$ and $R^{\prime}=R \backslash R_{1}$. For any $\left(i_{1}, \ldots, i_{q}\right) \in R_{1}$, by (3.2),

$$
f_{i_{1} \ldots i_{q}}=F\left(\varphi\left(v_{i_{1}}\right), \ldots, \varphi\left(v_{i_{q}}\right)\right)=\lambda_{i_{1}} \ldots \lambda_{i_{q}} f_{i_{1} \ldots i_{q}}+\sum_{\left(j_{1}, \ldots, j_{q}\right) \in R^{\prime}} c_{i_{1} \ldots i_{q}, j_{1} \ldots j_{q}} f_{j_{1} \ldots j_{q}},
$$

where each coefficient $c_{i_{1} \ldots i_{q}, j_{1} \ldots j_{q}}$ is a polynomial of $\lambda_{i}$ 's and $\gamma_{i j}$ 's. It then follows from $\lambda_{i_{1}} \ldots \lambda_{i_{q}} \neq 1$ that $f_{i_{1} \ldots i_{q}}$ can be expressed as a rational function of other variables appearing in (3.3). Note that the expression of this rational function does not depend on $F$. 
Now each $F_{i_{1} \ldots i_{q}}$ is a rational function in the variables $\tau_{i j}(1 \leq i, j \leq m), \lambda_{i}$ $(1 \leq i \leq l), \gamma_{i j}(1 \leq i \leq m-l, 1 \leq j \leq l)$ and $f_{i_{1} \ldots i_{q}}\left(\left(i_{1}, \ldots, i_{q}\right) \in R^{\prime}\right)$. The total number of variables used here is $m^{2}+l+(m-l) l+\left(\begin{array}{c}m \\ q\end{array}\right)-\left(\begin{array}{l}l \\ q\end{array}\right)$, which is less than $\left(\begin{array}{c}m \\ q\end{array}\right)$ for sufficiently large $m$ as $q \geq 3$ and $l=\left\lfloor\frac{m}{54}\right\rfloor$.

Thus by Lemma 3.6 there exists a non-zero polynomial $P$ in the variables $F_{i_{1} \ldots i_{q}}$, where $1 \leq i_{1}<\ldots<i_{q} \leq m$, such that $P=0$ for any special function $F$. Now $X_{m}$ can be chosen to be the zero set of $P$, which is obviously a proper closed set.

For the situation $F \in\left(\vee^{q} \mathbb{R}^{m}\right)^{*}$, we only need in the proof above to substitute $\left(\wedge q \mathbb{R}^{m}\right)^{*}$ by $\left(\vee^{q} \mathbb{R}^{m}\right)^{*}$, substitute $\left(\begin{array}{c}m \\ q\end{array}\right),\left(\begin{array}{c}l \\ q\end{array}\right)$ by $\left(\begin{array}{c}m+q-1 \\ q\end{array}\right),\left(\begin{array}{c}l+q-1 \\ q\end{array}\right)$, respectively, and change the restriction $i_{1}<\ldots<i_{q}$ to $i_{1} \leq \ldots \leq i_{q}$ in the definitions of $R$ and $R_{1}$.

\section{MAIN THEOREM}

In this section we will prove Theorem 1.1. As mentioned in the introduction, we only need to prove this when the target $W$ is oriented.

For a graded algebra $A=\bigoplus_{i=0}^{\infty} A_{i}$, over $\mathbb{Q}$, we always assume its multiplication $\cup$ is associative, $A_{0}=\mathbb{Q}$ and $1 \in \mathbb{Q}=A_{0}$ is the unit element of the algebra $A$. $A$ is called commutative if for all $a \in A_{r}, b \in A_{s}, a \cup b=(-1)^{r s}(b \cup a)$.

The rational cohomology ring $H^{*}(X ; \mathbb{Q})$ of a path-connected topological space $X$ is a commutative graded algebra over $\mathbb{Q}$ (cf. $[\mathrm{Sp}$, pp. 263-264).

Definition 4.1. Let $A=\bigoplus_{i=0}^{\infty} A_{i}$ be a commutative graded algebra over $\mathbb{Q}$. Assume that there exists a positive integer $n$ such that $A_{n} \cong \mathbb{Q}, A_{i}=0$ for $i>n$ and $A_{i}$ is finite dimensional for $i<n$. Let $\varphi_{i}$ be the map from $A_{i}$ to $\operatorname{Hom}\left(A_{n-i}, A_{n}\right)$ defined by $\varphi_{i}(u)(v)=u \cup v, \forall u \in A_{i}, v \in A_{n-i}$. We say that $A$ satisfies Poincaré duality if $\varphi_{i}$ is an isomorphism from $A_{i}$ to $\operatorname{Hom}\left(A_{n-i}, A_{n}\right)$ for all $0 \leq i \leq n$.

Proposition 4.2. Suppose that $p$ is a positive integer, and $q \geq 3$ is an odd integer. Let $F \in\left(\wedge^{q} V\right)^{*}$ if $p$ is odd and $F \in\left(\vee^{q} V\right)^{*}$ if $p$ even, where $V$ is a finite dimensional vector space over $\mathbb{Q}$.

There exists a commutative graded algebra $A=\bigoplus_{i=0}^{p q} A_{i}$ satisfying Poincaré duality such that

(i) $A_{p}=V, A_{p q}=\mathbb{Q}$, and $A_{i} \neq 0$ only if $i$ is a multiple of $p$,

(ii) for all $v_{1}, \ldots, v_{q} \in A_{p}=V, F\left(v_{1}, \ldots, v_{q}\right)=v_{1} \cup \ldots \cup v_{q}$.

Proof. First assume that $p$ is odd. Let $q=2 r+1$. We construct $A$ by setting

$$
A_{i}= \begin{cases}\wedge^{s} V & \text { if } i=s p \text { and } 0 \leq s \leq r, \\ \left(\wedge^{2 r+1-s} V\right)^{*} & \text { if } i=s p \text { and } r+1 \leq s \leq 2 r+1, \\ 0 & \text { otherwise. }\end{cases}
$$

Now suppose $u \in A_{s p}, v \in A_{t p}(0 \leq s, t \leq 2 r+1)$. We define $u \cup v$, the product of $u$ and $v$, as follows. (We assume that $s+t \leq 2 r+1$, since otherwise $u \cup v=0$.)

Case 1. If $s+t \leq r$, then $u \in A_{s p}=\wedge^{s} V, v \in A_{t p}=\wedge^{t} V$, and we define $u \cup v \in A_{(s+t) p}=\wedge^{s+t} V$ to be $u \wedge v$.

Case 2. If $s \leq r, t \leq r$ and $s+t \geq r+1$, then we define $u \cup v \in A_{(s+t) p}=$ $\left(\wedge^{2 r+1-s-t} V\right)^{*}$ by the equation $(u \cup v)(w)=F(u \wedge v \wedge w)$ for $w \in \wedge^{2 r+1-s-t} V$.

Case 3. If $s \leq r, t \geq r+1$ and $s+t \leq 2 r+1$, then $u \in A_{s p}=\wedge^{s} V, v \in A_{t p}=$ $\left(\wedge^{2 r+1-t} V\right)^{*}$, and we define $u \cup v \in A_{(s+t) p}=\left(\wedge^{2 r+1-s-t} V\right)^{*}$ by the equation $(u \cup v)(w)=v(w \wedge u)$ for $w \in \wedge^{2 r+1-s-t} V$. 
Case 4. If $s \geq r+1, t \leq r$ and $s+t \leq 2 r+1$, then $u \in A_{s p}=\left(\wedge^{2 r+1-s} V\right)^{*}, v \in$ $A_{t p}=\wedge^{t} V$, and we define $u \cup v \in \bar{A}_{(s+t) p}=\left(\wedge^{2 r+1-s-t} V\right)^{*}$ by the equation $(u \cup v)(w)=u(v \wedge w)$ for $w \in \wedge^{2 r+1-s-t} V$.

A routine calculation shows that this product makes $A$ a commutative graded algebra satisfying Poincaré duality and conditions (i) except that $A_{p q}=\mathbb{Q}^{*}$. By the canonical isomorphism $f: \mathbb{Q}^{*} \rightarrow \mathbb{Q}$ (i.e. for $w \in \mathbb{Q}^{*}, f(w)=w(1)$ ), we can identify $A_{p q}$ with $\mathbb{Q}$. Then it is easy to see that condition (ii) holds.

If $p$ is even, we can just replace all $\wedge$ in the above construction by $\vee$.

Proof of Theorem [1.1. Suppose such an $(n+1)$-manifold $W$ exists. Since $n$ is a composite number and is not a power of 2 , we have a factorization $n=p q$, where $p$ is an integer $\geq 2$ and $q$ is an odd integer $\geq 3$. By Proposition 3.3, we can select an integer $m \geq 3 \beta_{p+1}(W)$ such that all special functions $F$ sit inside a proper closed subset $X_{m}$ of $\left(\wedge^{q} \mathbb{R}^{m}\right)^{*}$ (when $p$ is odd) or $\left(\vee^{q} \mathbb{R}^{m}\right)^{*}$ (when $p$ is even). Since $X_{m}$ is proper closed, we can pick an $F \notin X_{m}$ such that $F\left(e_{i_{1}}, \ldots, e_{i_{q}}\right) \in \mathbb{Q}$ for any $1 \leq i_{1}, \ldots, i_{q} \leq m$.

Taking $V=\mathbb{Q}^{m}$, by Proposition 4.2, there exists a commutative graded algebra $A$ satisfying Poincaré duality and conditions (i) and (ii). $p \geq 2$ guarantees that $A_{1}=$ 0 . By Theorem (13.2) from $\underline{\mathrm{Su}}$, there exists a simply-connected, closed, smooth $n$-manifold $M$ such that the rational cohomology $\operatorname{ring} H^{*}(M ; \mathbb{Q})$ is isomorphic to $A$. Denote this isomorphism by $h_{\mathbb{Q}}$, and define $h_{\mathbb{R}}=h_{\mathbb{Q}} \otimes$ id $: H^{*}(M ; \mathbb{R})=$ $H^{*}(M ; \mathbb{Q}) \otimes_{\mathbb{Q}} \mathbb{R} \rightarrow A \otimes_{\mathbb{Q}} \mathbb{R}$. Clearly $h_{\mathbb{R}}$ gives isomorphisms $H^{p}(M ; \mathbb{R}) \rightarrow \mathbb{R}^{m}$ and $H^{n}(M ; \mathbb{R}) \rightarrow \mathbb{R}$. Condition (ii) in Proposition 4.2 can be transformed to

$$
F\left(h_{\mathbb{R}}\left(x_{1}\right), \ldots, h_{\mathbb{R}}\left(x_{q}\right)\right)=h_{\mathbb{R}}\left(x_{1} \cup \ldots \cup x_{q}\right) .
$$

Note that $\beta_{p}(M)=m \geq 3 \beta_{p+1}(W)$; hence $\frac{1}{2}\left(\beta_{p}(M)-\beta_{p+1}(W)\right) \geq \frac{m}{3}$. Since $M$ can be topologically flatly embedded into $W$, by Proposition 2.1, there exists a subspace $V$ of $H^{p}(M ; \mathbb{R})$ and a linear transformation $\varphi: V \rightarrow H^{p}(M ; \mathbb{R})$ with no fixed non-zero vectors such that $\operatorname{dim} V \geq \frac{1}{2}\left(\beta_{p}(M)-\beta_{p+1}(W)\right) \geq \frac{m}{3}$ and for any $x_{1}, \ldots, x_{q} \in V$,

$$
x_{1} \cup \ldots \cup x_{q}=\varphi\left(x_{1}\right) \cup \ldots \cup \varphi\left(x_{q}\right) .
$$

Define $U=h_{\mathbb{R}}(V), \psi: U \rightarrow \mathbb{R}^{m}$ to be $\psi=h_{\mathbb{R}} \circ \varphi \circ h_{\mathbb{R}}^{-1}$. Then $\operatorname{dim} U \geq \frac{m}{3}$ and $\psi: U \rightarrow \mathbb{R}^{m}$ is a linear map with no fixed non-zero vectors. By equations (4.1) and (4.2), for any $y_{1}, \ldots, y_{q} \in U$, we have $F\left(y_{1}, \ldots, y_{q}\right)=F\left(\psi\left(y_{1}\right), \ldots, \psi\left(y_{q}\right)\right)$. Thus $F$ is special. But $F \notin X_{m}$, a contradiction.

\section{EMBEDding OF 4-MANiFOLdS INTO 5-MANIFOLDS}

In this section we prove Theorem 1.2, Let us first recall some basic knowledge about the intersection forms of 4-manifolds. For any compact, connected, oriented 4-manifold $M$, the cup product

$$
\cup: H^{2}(M, \partial M) \times H^{2}(M, \partial M) \rightarrow H^{4}(M, \partial M),(\alpha, \beta) \mapsto \alpha \cup \beta
$$

gives a symmetric bilinear form $Q_{M}$ over $\mathbb{Z}$ on $H_{2}(M)$ through the isomorphisms $H^{2}(M, \partial M) \cong H_{2}(M)$ and $H^{4}(M, \partial M) \cong \mathbb{Z}$. Clearly, $Q_{M}(a, b)=0$ if $a$ or $b$ is a torsion element. So $Q_{M}$ descends to a symmetric bilinear form on $H_{2}(M) /$ Torsion $\cong$ $\mathbb{Z}^{r}$.

For a given integral symmetric bilinear form $Q$ on a finitely generated free abelian group $A$, the $\operatorname{rank} \operatorname{rk}(Q)$ of $Q$ is the dimension of $A$. Extend and diagonalize $Q$ 
over $A \otimes_{\mathbb{Z}} \mathbb{R}$; the number of positive entries and the number of negative entries are denoted by $b_{2}^{+}$and $b_{2}^{-}$respectively, and the difference $b_{2}^{+}-b_{2}^{-}$is the signature $\sigma(Q)$ of $Q$.

We say $Q$ is indefinite if both $b_{2}^{+}$and $b_{2}^{-}$are positive. We say $Q$ is even if $Q(a, a)$ is even for any $a \in A$ and odd otherwise. By choosing a basis for $A$, one can express $Q$ as a symmetric matrix. If $\operatorname{det} Q= \pm 1$, we say $Q$ is unimodular. From now on, we will not specify the basis for $A$ and just denote symmetric bilinear forms by symmetric matrices.

For the intersection form $Q_{M}$, its signature is defined to be the signature $\sigma(M)$ of the manifold $M$. It follows from Poincaré duality that $Q_{M}$ is unimodular if $M$ is closed.

Let $S$ and $S^{\prime}$ be two matrices that give integral symmetric bilinear forms on $A$ and $A^{\prime}$ through a basis for $A$ and a basis for $A^{\prime}$. The union of these two bases forms a basis for $A \oplus A^{\prime}$. Under this basis, the matrix $\operatorname{diag}\left(S, S^{\prime}\right)$ gives a bilinear form on $A \oplus A^{\prime}$, which is denoted by $S \oplus S^{\prime}$. For simplicity, $S \oplus S \oplus \ldots \oplus S$, which contains $r$ copies of $S$, is denoted by $r S$. Note that $Q_{M} \oplus Q_{M^{\prime}}$ is the intersection form of $M \# M^{\prime}$ for closed 4-manifolds $M$ and $M^{\prime}$.

The following lemma tells us that any simply-connected indefinite closed 4manifold can be built up by a number of fixed 4-manifolds through the connected sum operation. Let \#rM be the connected sum of $r$ copies of $M$ for an integer $r>0$ and $\# 0 M^{4}=S^{4}$.

Lemma 5.1. There exist oriented closed connected 4 -manifolds $M_{i}, 1 \leq i \leq 7$, such that for any simply-connected closed 4-manifold $M$ with indefinite intersection form, $M$ is homeomorphic to \# $k_{1} M_{1} \# k_{2} M_{2} \# \ldots \# k_{7} M_{7}$ for some non-negative integers $k_{i}$.

Proof. All the indefinite unimodular forms can be classified as follows (cf. GS], Theorem 1.2.21). If $Q_{M}$ is odd, then it is isomorphic to $b_{2}^{+}[+1] \oplus b_{2}^{-}[-1]$. If $Q_{M}$ is even, then it is isomorphic to $c_{1} E_{8} \oplus c_{2} H$, where $c_{1}, c_{2}$ are integers, $c_{2} \geq 0, E_{8}$ is an even form with $\operatorname{rk}\left(E_{8}\right)=\sigma\left(E_{8}\right)=8$, and $H=\left[\begin{array}{ll}0 & 1 \\ 1 & 0\end{array}\right]$.

In $[\mathrm{Fr}$, Freedman proved the classification result for simply-connected 4-manifolds as follows. For every integral, unimodular symmetric bilinear form $Q$, there exists a simply-connected, closed, topological 4-manifold $M$ such that $Q_{M} \cong Q$. If $Q$ is even, this manifold is unique up to homeomorphism. If $Q$ is odd, there are exactly two different homeomorphism types of such manifolds. At most one of these two homeomorphism types carries a smooth structure.

Now we need to consider two cases.

$\underline{\text { Case 1. }} Q_{M}$ is odd, i.e. $Q_{M} \cong p[+1] \oplus q[-1]$.

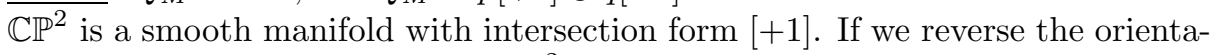
tion of $\mathbb{C P}^{2}$, the resulting manifold $\overline{\mathbb{C P}}^{2}$ has intersection form $[-1]$. By Freedman's theorem above, there are also non-smoothable manifolds $X_{1}$ and $X_{-1}$ that have intersection forms $[+1]$ and $[-1]$, respectively.

Note that the two manifolds with the same odd intersection form can be distinguished by Kirby-Siebenmann triangulation invariants $\mathrm{KS} \in \mathbb{Z}_{2}$ (cf. [KS]). One has non-zero invariant and cannot be smooth, while the other has zero invariant and is stably smoothable. Thus $\operatorname{KS}\left(X_{1}\right)=\operatorname{KS}\left(X_{-1}\right)=1$.

The Kirby-Siebenmann invariant is additive in the sense that (cf. FQ, Section $10.2 \mathrm{~B})$ if there is a homeomorphism of boundaries $\partial M \cong \partial N$, then 
$\mathrm{KS}\left(M \cup_{\partial} N\right)=\mathrm{KS}(M)+\mathrm{KS}(N)$. Combined with the fact that $D^{4}$ is smoothable, we know that $\operatorname{KS}\left(M_{1} \# M_{2}\right)=\operatorname{KS}\left(M_{1}\right)+\operatorname{KS}\left(M_{2}\right)$ for connected, closed, topological 4-manifolds $M_{1}$ and $M_{2}$.

Clearly the form $p[+1] \oplus q[-1]$ can be realized by both $X_{1} \#(p-1) \mathbb{C P}^{2} \# q \overline{\mathbb{C P}}^{2}$ (if $p>0$ ) and $X_{-1} \# p \mathbb{C P}^{2} \#(q-1) \overline{\mathbb{C P}}^{2}$ (if $q>0$ ). These two manifolds have nontrivial Kirby-Siebenmann invariant and are therefore non-smoothable. On the other hand, the smooth manifold $\# p \mathbb{C P}^{2} \# q \overline{\mathbb{C P}}^{2}$ also has intersection form $p[+1] \oplus q[-1]$.

Now no matter whether $M$ is smooth or not, it must be homeomorphic to the connected sum of some copies of $M_{1}=\mathbb{C P}^{2}, M_{2}=\overline{\mathbb{C P}}^{2}, M_{3}=X_{1}$ and $M_{4}=X_{-1}$.

Case 2. $Q_{M}$ is even, i.e. $Q_{M} \cong p E_{8} \oplus q\left(-E_{8}\right) \oplus r H$, where $p, q$ and $r$ are non-negative integers.

Let $X_{E_{8}}$ and $X_{-E_{8}}$ be the simply-connected 4-manifolds with intersection forms $E_{8}$ and $-E_{8}$, respectively. Clearly $S^{2} \times S^{2}$ has intersection form $H$. Then $p X_{E_{8}} \#$ $q X_{-E_{8}} \# r\left(S^{2} \times S^{2}\right)$ has the same intersection form as $M$, and by Freedman's theorem, they are homeomorphic.

Therefore, we can finish the proof by taking $M_{5}=X_{E_{8}}, M_{6}=X_{-E_{8}}$, and $M_{7}=S^{2} \times S^{2}$.

When $M$ is a 4-manifold with boundary, we can glue $M$ and $-M$ together by the identity map between $\partial M$ and $\partial(-M)$. The resulting manifold is called the double of $M$ and denoted by $D M$.

Lemma 5.2. For any simply-connected, compact topological 4-manifold $M$ with $\partial M \neq \emptyset$, its double DM is homeomorphic to either $\# k \mathbb{C P}^{2} \# k \overline{\mathbb{C P}}^{2}$ or $\# k S^{2} \times S^{2}$ for some non-negative integer $k$.

Proof. Clearly $D M$ is a simply-connected, closed 4-manifold with $\sigma(D M)=\sigma(M)+$ $\sigma(-M)=0$ (cf. [Ki], Chapter II, Theorem 5.3). By the classification result of symmetric bilinear forms (cf. the first paragraph in the proof of Lemma 5.1), the intersection form $Q$ of $D M$ is either $k[+1] \oplus k[-1]$ or $k H$, depending on whether $Q$ is odd or even.

When $Q$ is odd, $D M$ and the smooth manifold $\# k \mathbb{C P}^{2} \# k \overline{\mathbb{C P}}^{2}$ have the same intersection form. As $\mathrm{KS}(D M)=\mathrm{KS}(M)+\mathrm{KS}(-M)=0$, they are homeomorphic. When $Q$ is even, $D M$ and $\# k S^{2} \times S^{2}$ have the same intersection form and are therefore homeomorphic.

The next lemma is the key observation in proving Theorem 1.2 .

Lemma 5.3. For any oriented closed connected 4-manifold $M$, there exists an oriented closed connected 5-manifold $W$ such that for any positive integer $r$, \#rM can be topologically flatly embedded into $W$.

Proof. First let us recall the definition of surgery operations. (For details, see GS.) Let $M$ be a closed $n$-manifold and let $\varphi: S^{k} \times D^{n-k} \rightarrow M(0 \leq k \leq n)$ be an embedding. We can remove $\varphi\left(\operatorname{int}\left(S^{k} \times D^{n-k}\right)\right)$ from $M$ and replace it with $D^{k+1} \times S^{n-k-1}$ by gluing along $\varphi \mid S^{k} \times S^{n-k-1}$. This procedure is called a surgery by $\varphi$ on $M$. Clearly, the new manifold is still closed.

Choose an embedding $\varphi: S^{0} \times D^{4} \rightarrow M^{4}$, and denote the images of $\varphi$, which are two disjoint 4-balls, by $D^{\prime}$ and $D^{*}$ respectively. Set $M^{o}=M-\operatorname{int}\left(D^{\prime} \cup D^{*}\right)$. Then the surgery by $\varphi$ on $M$ gives a manifold $M^{\prime}=M^{o} \cup S^{3} \times D^{1}$. Let $p: S^{3} \times D^{1} \rightarrow D^{1}$ be the projection onto the second factor. From now on, we regard $D^{1}$ as $[0,1]$ and 


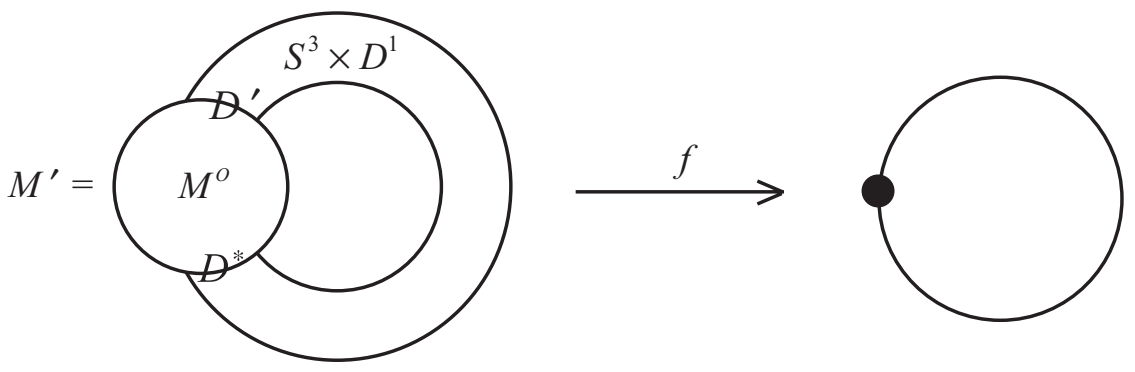

Figure 1

$S^{1}$ as $\mathbb{R} / \mathbb{Z}$. Define $q: D^{1} \rightarrow S^{1}$ to be $q(x)=[x]$. Clearly $q \circ p$ can be extended to a map $f: M^{\prime} \rightarrow S^{1}$ as we can let the whole $M^{o}$ map to [0] in $S^{1}$. Figure 1 illustrates $M^{\prime}$ and the map $f$, where $M^{o}$ maps to the big dot on the circle.

Let $W^{\prime}=M^{\prime} \times S^{1}$, and $g: W^{\prime} \rightarrow T^{2}=S^{1} \times S^{1}$ be defined by $g(x, y)=(f(x), y)$. Choose a small closed disk $D$ in $T^{2}$ centered at $\left(\left[\frac{1}{4}\right],[0]\right)$ and identify it with the standard 2-ball $D^{2}$. Then there is a homeomorphism from $g^{-1}(D)$ to $S^{3} \times D^{2}$. Thus we can apply a surgery operation which cuts out $\operatorname{int}\left(g^{-1}(D)\right)$ from $W^{\prime}$ and glues back $D^{4} \times S^{1}$. We will show that the resulting manifold $W$ has the desired property.

Let $h: W \rightarrow T^{2}$ be given by $g$ on $W^{\prime}-\operatorname{int}\left(g^{-1}(D)\right)$ and the projection onto the factor $\partial D=S^{1}$ in the piece $D^{4} \times S^{1}$. For a point $q \in T^{2}$, we have $h^{-1}(q)=M^{o}$ if $q=([0],[y]), h^{-1}(q)=\varnothing$ if $q \in \operatorname{int}(D), h^{-1}(q)=D^{4}$ if $q \in \partial D$ and $h^{-1}(q)=S^{3}$ otherwise.

For any positive integer $r$, define $C_{r}=\left\{\left(\left[\frac{1}{4}+r t\right],[t]\right) \mid 0 \leq t \leq 1\right\} \subset T^{2}$ and $X_{r}=h^{-1}\left(C_{r}\right)$. We want to show that $X_{r}$ is $\# r M$. The $T^{2}$ along with the curve $C_{r}$ are shown in Figure 2, where the shaded region denotes the disk $D$.

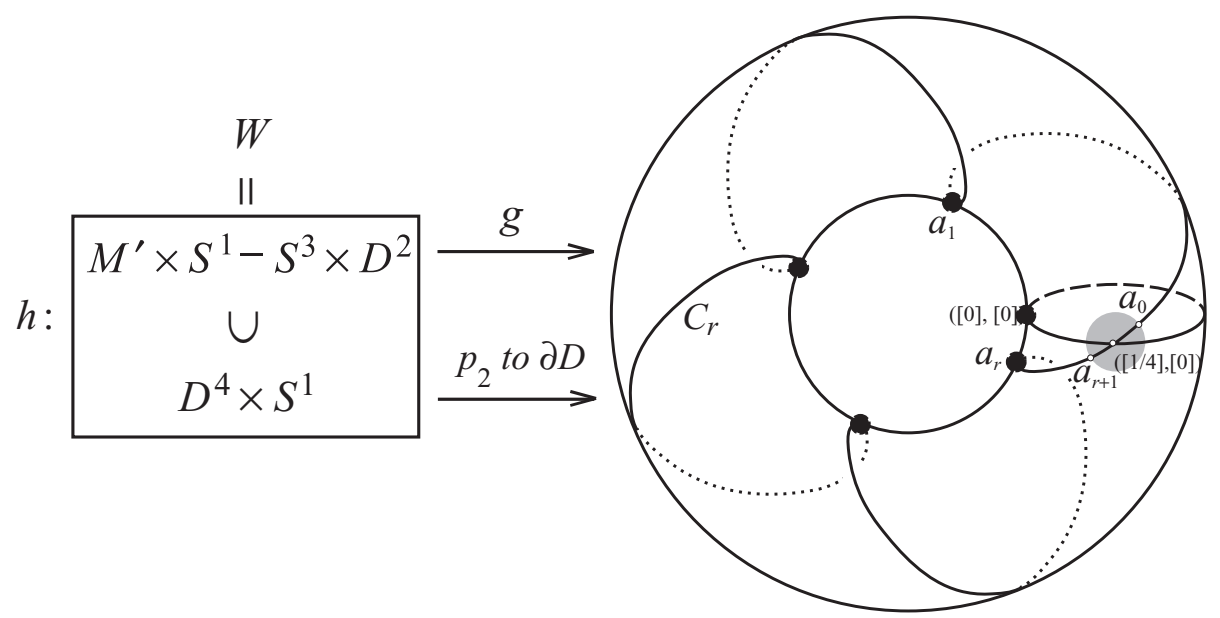

Figure 2

The curve $C_{r}$ intersects the longitude $\{([0],[y]) \mid 0 \leq y \leq 1\}$ in $r$ points, say successively $a_{1}, \cdots, a_{r}$, as $t$ increases. Name the two points of $C_{r} \cap \partial D$ by $a_{0}$ and 
$a_{r+1}$. Note that $h^{-1}\left(a_{i}\right)(1 \leq i \leq r)$ is a copy of $M^{o}$, both $h^{-1}\left(a_{0}\right)$ and $h^{-1}\left(a_{r+1}\right)$ are $D^{4}$, while for each sub-arc of $C_{r}$ that connects $a_{i}$ and $a_{i+1}(0 \leq i \leq r)$, its pre-image under $h$ is a copy of $S^{3} \times D^{1}$. Figure 3 illustrates $X_{r}$.

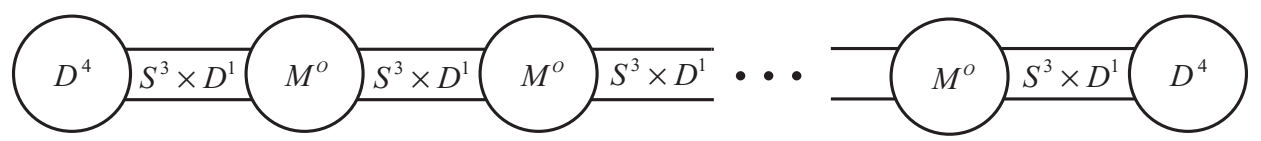

Figure 3

For the given manifold $M$, if $M-\operatorname{int}\left(D^{\prime}\right)$ and $M-\operatorname{int}\left(D^{*}\right)$ are glued to $S^{3} \times I$ along the orientation reversing homeomorphism $\psi_{1}: \partial D^{\prime} \rightarrow-\left(S^{3} \times\{0\}\right)$ and $\psi_{2}: \partial D^{*} \rightarrow S^{3} \times\{1\}$, respectively, then one gets a closed manifold $N$. In fact, $N$ is just $M \# M$ as $S^{3} \times\left\{\frac{1}{2}\right\}$ cuts $N$ into two components which are homeomorphic to $M-\operatorname{int}\left(D^{\prime}\right)$ and $M-\operatorname{int}\left(D^{*}\right)$, respectively. It can be seen from Figure 3 that the adjacent copies of $M^{o}$ are connected to $S^{3} \times D^{1}$ in the manner described by $\psi_{1}$ and $\psi_{2}$ above, while the two $D^{4}$ fill the holes of $D^{*}$ and $D^{\prime}$ in the first and the last copies of $M^{o}$. Therefore $X_{r}$ is homeomorphic to \#rM.

Choose a very small $\varepsilon>0$ and set $N=\left\{\left(\left[\frac{1}{4}+r t\right],[t+s]\right) \mid 0 \leq t \leq 1,-\varepsilon \leq s \leq\right.$ $\varepsilon\} \subset T^{2}$. All the points in $N$ with fixed $s$ form a curve $C_{r}^{s}$ in $T^{2}$. The above argument for $C_{r}$ applies to this curve as well. So $h^{-1}\left(C_{r}^{s}\right)$ is still \#rM. Note that $h^{-1}\left(C_{r}^{s}\right)$ vary continuously on $s$. Therefore $h^{-1}(N)$ gives an embedding of $(\# r M) \times[-\varepsilon, \varepsilon]$ in $W$.

Lemma 5.4. If there are topologically flat embeddings $M_{1}^{4} \hookrightarrow W_{1}^{5}$ and $M_{2}^{4} \hookrightarrow W_{2}^{5}$, then $M_{1} \# M_{2}$ can be topologically flatly embedded into $W_{1} \# W_{2}$.

Proof. Choose $D_{i}^{4}$ in $M_{i}$. Since $M_{i} \times I$ is a part of $W_{i}, D_{i}^{4} \times I$ is a 5 -ball in $W_{i}$ $(i=1,2)$. Cut out these two 5 -balls from the $W_{i}$, respectively, and glue their boundaries. Then we have an embedding of $\left(M_{1} \# M_{2}\right) \times I$ into $W_{1} \# W_{2}$.

Finally we are ready to prove Theorem 1.2 .

Proof. (a) Choose $M$ to be $M_{i}, 1 \leq i \leq 7$ respectively as given in Lemma 5.1 and construct corresponding 5-manifolds $W_{i}$ by Lemma 5.3. It follows from Lemma 5.4 that the desired manifold $W$ can be chosen to be $W_{1} \# W_{2} \# \ldots \# W_{7}$.

(b) Clearly $M$ embeds in $D M$.

It is well known (cf. GS, Section 4.6) that $\mathbb{C P}^{2} \# \overline{\mathbb{C P}}^{2} \cong S^{2} \tilde{\times} S^{2}$ and that $S^{2} \tilde{\times} S^{2} \# S^{2} \tilde{\times} S^{2} \cong S^{2} \times S^{2} \# S^{2} \tilde{\times} S^{2}$, where $S^{2} \tilde{\times} S^{2}$ denotes the unique non-trivial $S^{2}$-bundle over $S^{2}$. Combined with Lemma 5.2, $D M$ is homeomorphic to either $\# k S^{2} \times S^{2}$ or $\#(k-1) S^{2} \times S^{2} \# S^{2} \tilde{x} S^{2}$.

$S^{2} \tilde{\times} S^{2}$ can be embedded into $S^{2} \tilde{\times} S^{3}$ because the inclusion $\mathrm{SO}(3) \hookrightarrow \mathrm{SO}(4)$ induces the isomorphism $\pi_{1}(\mathrm{SO}(3)) \cong \pi_{1}(\mathrm{SO}(4)) \cong \mathbb{Z}_{2}$. Also, $S^{2} \times S^{2}$ can be embedded into $\mathbb{R}^{5}$ through the standard inclusion $S^{2} \times D^{3} \hookrightarrow \mathbb{R}^{5}$. Therefore by Lemma 5.4, both $\# k S^{2} \times S^{2}$ and $\#(k-1) S^{2} \times S^{2} \# S^{2} \tilde{\times} S^{2}$ can be embedded into $S^{2} \tilde{\times} S^{3}$. 


\section{REFERENCES}

[Di] F. Ding, Smooth structures on some open 4-manifolds, Topology 36 (1997), no. 1, 203-207. MR:1410471 (97g:57040)

[EL] A. L. Edmonds, C. Livingston, Embedding punctured lens spaces in four-manifolds, Comment. Math. Helv. 71 (1996), no. 2, 169-191. MR.1396671 (97h:57049)

[Fa1] F. Fang, Embedding 3-manifolds and smooth structures of 4-manifolds, Topology Appl. 76 (1997), no. 3, 249-259. MR1441755 (98b:57037)

[Fa2] F. Fang, Smooth structures on $\Sigma \times \mathbb{R}$, Topology Appl. 99 (1999), no. 1, 123-131. MR1720366 (2001a:57044)

[Fr] M. H. Freedman, The topology of four-dimensional manifolds, J. Differential Geom. 17 (1982), no. 3, 357-453. MR679066 (84b:57006)

[FQ] M. H. Freedman, F. Quinn, Topology of 4-manifolds, Princeton University Press, Princeton, NJ, 1990. MR 1201584 (94b:57021)

[GS] R. E. Gompf, A. I. Stipsicz, 4-manifolds and Kirby calculus, Graduate Studies in Math. 20, Amer. Math. Soc., Providence, RI, 1999. MR.1707327 (2000h:57038)

[Hi] M. W. Hirsch, The imbedding of bounding manifolds in Euclidean space, Ann. of Math. (2) 74 (1961), 494-497. MR0133136 (24:A2970)

[HJ] R. A. Horn, C. R. Johnson, Matrix analysis, Cambridge University Press, Cambridge, 1990. MR:1084815 (91i:15001)

[Ka] A. Kawauchi, The imbedding problem of 3-manifolds into 4-manifolds, Osaka J. Math. 25 (1988), no. 1, 171-183. MR937194 (89g:57042)

[Ki] R. C. Kirby, The topology of 4-manifolds, Lecture Notes in Math. 1374, Springer-Verlag, Berlin, 1989. MR1001966 (90j:57012)

[KS] R. C. Kirby, L. C. Siebenmann, Foundational essays on topological manifolds, smoothings, and triangulations, Ann. of Math. Studies 88, Princeton University Press, Princeton, NJ, 1977. MR.0645390 (58:31082)

[La] S. Lang, Algebra, Graduate Texts in Math. 211, Springer-Verlag, New York, 2002. MR1878556 (2003e:00003)

[Ro] V. A. Rohlin, The embedding of non-orientable three-manifolds into five-dimensional Euclidean space, Soviet Math. Dokl. 6 (1965), 153-156. MR0184246 (32:1719)

[Sh] R. Shaw, Linear algebra and group representations, Vol. II, Academic Press, London-New York, 1983. MR701854 (84m:15003)

[Shi] T. Shiomi, On imbedding 3-manifolds into 4-manifolds, Osaka J. Math. 28 (1991), no. 3, 649-661. MR 1144478 (92m:57028)

[Sp] E. H. Spanier, Algebraic topology, Springer-Verlag, New York, 1966. MR666554 (83i:55001)

[Su] D. Sullivan, Infinitesimal computations in topology, Inst. Hautes Études Sci. Publ. Math. 47 (1977), 269-331 (1978). MR0646078 (58:31119)

[Wa] C. T. C. Wall, All 3-manifolds imbed in 5-space, Bull. Amer. Math. Soc. 71 (1965), 564-567. MR0175139 (30:5324)

[WZ] S. Wang, Q. Zhou, How to embed 3-manifolds into 5-space, Adv. in Math. (China) 24 (1995), no. 4, 309-312. MR1358889

Lmam and School of Mathematical Sciences, Peking University, Beijing 100871, PeoPLE'S REPUBLIC OF CHINA

E-mail address: dingfan@math.pku.edu.cn

LMam and School of Mathematical Sciences, Peking University, Beijing 100871, PeoPLE'S REPUBLIC OF CHINA

E-mail address: wangsc@math.pku.edu.cn

Department of Mathematics, University of California at Berkeley, Berkeley, CalIFORNIA 94720

E-mail address: jgyao@math.berkeley.edu 